\title{
The extended chromosphere of CoRoT-2A *
}

\section{Discovery and analysis of the chromospheric Rossiter-McLaughlin effect}

\author{
S. Czesla ${ }^{1}$, S. Schröter ${ }^{1}$, U. Wolter ${ }^{1}$, C. von Essen ${ }^{1}$, K. F. Huber ${ }^{1}$, J. H. M. M. Schmitt ${ }^{1}$, \\ D. E. Reichart ${ }^{2}$, and J. P. Moore $^{2}$

\begin{abstract}
1 Hamburger Sternwarte, Universität Hamburg, Gojenbergsweg 112, 21029 Hamburg, Germany e-mail: stefan.czesla@hs.uni-hamburg.de

2 Department of Physics and Astronomy, University of North Carolina, Chapel Hill, CB 3255, Phillips Hall, Chapel Hill, NC 27599-3255, USA
\end{abstract}

Received 7 September 2011 / Accepted 16 January 2012

\section{ABSTRACT}

\begin{abstract}
The young G7V dwarf CoRoT-2A is transited by a hot Jupiter and among the most active planet host-stars known to date. We report on the first detection of a chromospheric Rossiter-McLaughlin effect observed in the $\mathrm{Ca}$ II $\mathrm{H}$ and $\mathrm{K}$ emission-line cores. In Ca II $\mathrm{H}$ and $\mathrm{K}$, the transit lasts $15 \%$ longer than that observed in visual photometry, indicating that chromospheric emission extends $100000 \mathrm{~km}$ beyond the photosphere. Our analysis is based on a time series of high-resolution UVES spectra obtained during a planetary transit and simultaneously obtained photometry observed with one of the PROMPT telescopes. The chromospheric Rossiter-McLaughlin effect provides a new tool to spatially resolve the chromospheres of active planet host-stars.
\end{abstract}

Key words. stars: individual: CoRoT-2A - planetary systems - stars: late-type

\section{Introduction}

The G7V dwarf CoRoT-2A is one of the most active planet hoststars known to date. Every $1.74 \mathrm{~d}$, the star is transited by the unusually inflated hot Jupiter CoRoT-2b (Alonso et al. 2008; Bouchy et al. 2008). CoRoT-2A is young (100-300 Ma) and has basically solar metallicity (Schröter et al. 2011). It shows a high level of magnetic activity as diagnosed from the amplitude of its photometric variation, strong chromospheric emission lines, and coronal X-ray emission (Alonso et al. 2008; Knutson et al. 2010; Schröter et al. 2011). Furthermore, Schröter et al. (2011) provided strong evidence that CoRoT-2A's optical neighbor, 2MASS J19270636+0122577, is a true physical companion of spectral type K9, as earlier hypothesized by Alonso et al. (2008), making CoRoT-2 a wide binary system with at least one planet.

The CoRoT-2 system has been subject to a multitude of photometric and spectroscopic studies. To name but a few: Alonso et al. (2008) reported the discovery of CoRoT-2b and first radial velocity (RV) measurements, later refined by Bouchy et al. (2008); Gillon et al. (2010) studied the secondary transit observed with Spitzer's IRAC instrument and Snellen et al. (2010) analyzed the secondary transit as observed by CoRoT; Lanza et al. (2009), Czesla et al. (2009), and Huber et al. (2009, 2010) used light-curve inversion techniques to recover CoRoT-2A's surface; finally, Ammler-von Eiff et al. (2009) and Schröter et al. (2011) investigated the spectral properties, activity, and X-ray emission of the system.

During a transit, the planet occults different parts of the rotating stellar surface, which leads to the Rossiter-McLaughlin effect (RME). The occultation introduces asymmetries into the

\footnotetext{
* Based on observations obtained with UVES at the ESO VLT Kueyen telescope (program ID 385.D-0426).
}

rotationally broadened line profile, which result in an apparent radial velocity (RV) shift of the stellar spectrum. The RME, first observed in eclipsing binary stars (Schlesinger 1910; Rossiter 1924; McLaughlin 1924), has become a standard tool to analyze the geometry of exoplanetary systems (e.g., Fabrycky \& Winn 2009). Analytical models of this effect, based on the first moment of spectral lines, have been presented by Ohta et al. (2005) and Giménez (2006) and are commonly applied to modeling, although they may not exactly reproduce the results obtained by cross-correlation techniques (e.g., Triaud et al. 2009; Hirano et al. 2010).

The RME has widely been used to measure the projected rotation velocity of planet host-stars and the alignment of their rotation axis relative to the planetary orbit (e.g., Bouchy et al. 2008). Snellen (2004) and Dreizler et al. (2009) proposed the use of the RME to probe the planetary atmosphere by analyzing the differences between measurements in various spectral lines showing potentially large absorption due to the planet's exosphere. In contrast to narrow-band photometry, the differential RME measurement does not rely on accurate photometric calibration (Snellen 2004).

The applicability of the RME is not limited to the study of the planetary orbit and atmosphere, but can be extended to the study of the stellar atmosphere. By confining the RME measurement to spectral lines originating in individual layers of the stellar atmosphere, the properties of these layers can be separately analyzed. In this paper, we demonstrate the feasibility of this approach for the stellar chromosphere. While the apparent surface brightness of the photosphere decreases toward the limb, the optically thin chromosphere and corona show limb-brightening (Wolter \& Schmitt 2005). Narrow-band transit light-curves of limb-brightened emission lines are expected to display characteristic profiles (Assef et al. 2009; Schlawin et al. 2010), which 
also applies to the RME derived from chromospheric emission lines. Therefore, the RME observed in chromospheric emission lines allows us to probe the spatial structure of the chromosphere, including both the chromospheric center-to-limb variation and inhomogeneities due to active regions. These measurements can be used to study the chromospheres of active planet host-stars and examine whether faculae dominate over spots on the surfaces of young, active stars, as found by Radick et al. (1998).

In this paper, we present time-resolved transit spectroscopy of CoRoT-2A obtained with the UVES spectrograph and simultaneous photometry obtained with the "Panchromatic Robotic Optical Monitoring and Polarimetry Telescopes" (PROMPT, Reichart et al. 2005). We refine the wavelength calibration of the spectra and discuss the RME observed in photospheric lines. In Sect. 3.3, we report the first detection of the prolonged RME in the chromospheric emission-line cores of $\mathrm{Ca}$ II $\mathrm{H}$ and $\mathrm{K}$, before closing with a discussion of our findings.

\section{Observation}

We acquired 24 high-resolution spectra of CoRoT-2A using the UVES spectrograph (Dekker et al. 2000) mounted at the VLT Kueyen. The observations were obtained on June 7, 2010, under program-ID 385.D-0426(A) and cover a full planetary transit of CoRoT-2b, including one hour before and two hours after the actual transit; the spectra have individual exposure times of $800 \mathrm{~s}$.

The UVES spectrograph was set up in dichroic 2 mode with a slit width of 0.7 arcsec. For the selected setup, UVES provides a wavelength coverage of 3800-9000 $\AA$ with gaps at 5000-5700 $\AA$ and 7500-7680 $\AA$ at a resolving power of about 60000 . Owing to the worsening seeing conditions during the second half of the night, we inserted the image slicer into the light path to reduce light losses during exposures Nos. 12-24. To reduce the UVES echelle data, we applied the REDUCE package developed by Piskunov \& Valenti (2002); for a more detailed discussion of the data and the reduction, we refer the reader to Schröter et al. (2011).

The transit observed with UVES was simultaneously followed by one of the six $0.41 \mathrm{~m}$ Ritchey-Chrétien telescopes belonging to the PROMPT observatory (Reichart et al. 2005) located at the "Cerro Tololo Inter-American Observatory" (CTIO) in Chile. The telescope is equipped with an "Apogee Alta U47" detector with a $1 k \times 1 k$ pixel array with a gain of 4.91 electrons/ADU and a read-out noise of 10 electrons.

A total of 528 science frames were taken with a fixed exposure time of $30 \mathrm{~s}$. No filter was used in the observations. We calibrated all science frames using a master bias, but refrained from applying a sky-flat correction, because the sky-flat exposures showed a strong illumination gradient from the center to the periphery, possibly caused by the projected shadow of the shutter. The following reduction was carried out using standard routines from the IRAF daophot package for image processing.

\section{Analysis}

We present an analysis of the temporal evolution of the observed spectra including a detailed presentation of the wavelength calibration, which is crucial to our analysis. All line profile and model fits as well as the Markov-chain Monte Carlo (MCMC) calculations make extensive use of routines of our

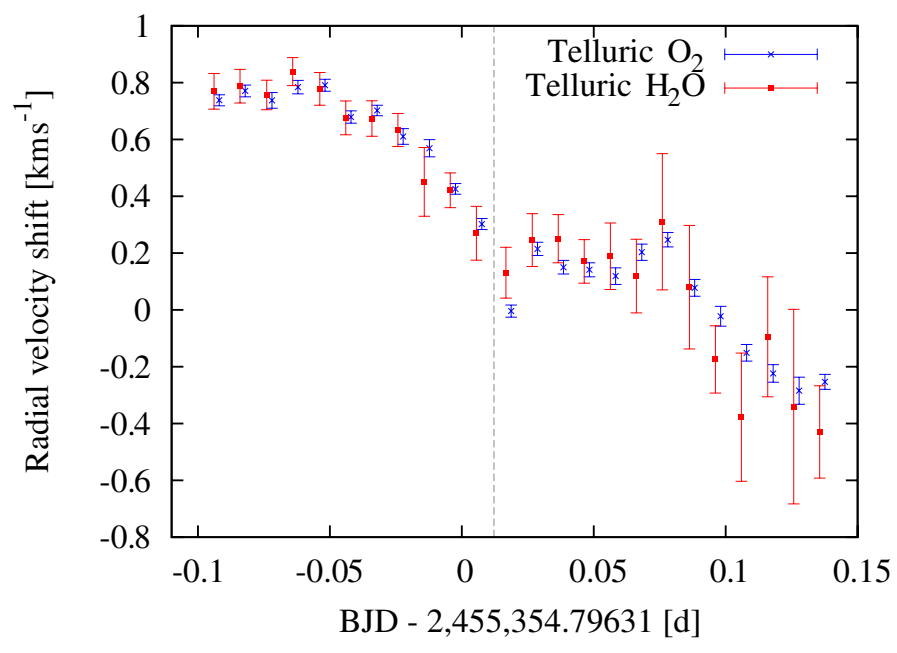

Fig. 1. Time-dependent drift of the UVES wavelength calibration determined from 64 isolated $\mathrm{H}_{2} \mathrm{O}$ (red) and $\mathrm{O}_{2}$ (blue) telluric absorption lines (small temporal displacement for clarity). The dashed line indicates the insertion of the image slicer.

PyAstronomy ${ }^{1}$ collection of Python packages, which provide a convenient interface to fitting and sampling algorithms implemented in the PyMC (Patil et al. 2010) and SciPy (Jones et al. 2001) packages.

\subsection{Refining the wavelength calibration using telluric standards}

Our wavelength calibration was obtained using Th-Ar lamp spectra. Since those spectra are not taken simultaneously with the science spectra, they cannot account for instrumental wavelength drifts produced, for example, by environmental effects. To obtain a more accurate, time-dependent wavelength calibration, we exploited telluric absorption lines. The power of this technique has already been demonstrated, for instance, by Snellen (2004), who used atmospheric $\mathrm{H}_{2} \mathrm{O}$ lines in a UVES spectrum to show that a precision of $\sim 5-10 \mathrm{~m} \mathrm{~s}^{-1}$ can be attained, and by Figueira et al. (2010), who found that the accuracy obtained by using atmospheric features can compete with that reached with gas-cell methods on timescales of days.

To obtain an absolute wavelength calibration, we determined the wavelengths of the strong atmospheric lines of $\mathrm{H}_{2} \mathrm{O}$ and $\mathrm{O}_{2}$. Telluric absorption lines of $\mathrm{H}_{2} \mathrm{O}$ are present around $6500 \AA$ and in the 6900-7400 $\AA$ region, while those pertaining to $\mathrm{O}_{2}$ populate the regions 6200-6300, 6800-6950, and 7650-7700. .

We selected the 64 strongest, isolated telluric lines $\left(27 \mathrm{H}_{2} \mathrm{O}\right.$, $37 \mathrm{O}_{2}$ ) and fitted them with Gaussians. The best-fit wavelengths were then compared with the spectral atlas of telluric absorption lines provided by the "high-resolution transmission molecular absorption database" (HITRAN ${ }^{2}$; Rothman et al. 2009). The sample standard deviation between observed and nominal wavelengths was used as an error estimate. Errors were determined for $\mathrm{H}_{2} \mathrm{O}$ and $\mathrm{O}_{2}$ separately and found to be larger for the $\mathrm{H}_{2} \mathrm{O}$ lines, which predominantly populate the wavelength band beyond $7000 \AA$, where the signal-to-noise (S/N) ratio decreases.

In Fig. 1, we show the thus obtained apparent RV shift of the 24 spectra. Clearly, the difference between nominal and

\footnotetext{
${ }^{1}$ http://www.hs.uni-hamburg.de/DE/Ins/Per/Czesla/PyA/ PyA/index.html

2 http://www.cfa.harvard.edu/hitran
} 
observed wavelength of the telluric lines follows a systematic, time-dependent trend. The blue shift corresponds to a drifting of approximately $1 \mathrm{~km} \mathrm{~s}^{-1}$ in five hours. The drift is neither linear nor monotonic, but shows a humpy behavior. Most notably, there is a sudden jump of $0.2 \mathrm{~km} \mathrm{~s}^{-1}$ after the 12 th observation. We find no wavelength-dependence of the trend in the 6200-7700 $\AA$ range covered by telluric lines.

This telluric drift may be caused by instrumental and atmospheric effects. As an evolving velocity field in the atmosphere gives rise to a Doppler shift, it induces a wavelengthproportional line shift. To check whether this is the case, we determined the apparent shift of telluric lines in seven echelle orders. Our analysis suggests that the drift of telluric lines does not show the characteristics of a Doppler shift, but is dominated by a displacement of the spectrum on the detector (about 0.1 CCD pixels at $6700 \AA$ ).

The telluric line drift may be the result of a changing dispersion relation caused by a variation in the ambient conditions. The ambient pressure and temperature reported in the FITS headers, indeed, follow a pattern reminiscent of the telluric line drift. Figure 2 shows both quantities as a function of time. Ambient pressure and temperature are both strongly correlated with the telluric drift pattern yielding Pearson correlation coefficients of 0.92 and 0.80 , corresponding to shifts of $1.38 \mathrm{~km} \mathrm{~s}^{-1} / \mathrm{hPa}$ and $1.12 \mathrm{~km} \mathrm{~s}^{-1} /{ }^{\circ} \mathrm{C}$. According to the ESO UVES User Manual (Kaufer et al. 2011) and the ESO Quality Control Group (2005), variations in temperature yield shifts of typically 0.35 pixels $/{ }^{\circ} \mathrm{C}\left(0.5 \mathrm{~km} \mathrm{~s}^{-1} /{ }^{\circ} \mathrm{C}\right)$ in dispersion direction and changes in the ambient pressure cause typical shifts of 0.05 pixels $/ \mathrm{hPa}\left(0.1 \mathrm{~km} \mathrm{~s}^{-1} / \mathrm{hPa}\right)$. Given the environmental conditions during the night (see Fig. 2), temperature variations dominate the instrumental drift.

Although the observed drift in our spectra is larger than that inferred from the UVES manual, we identify the ambient conditions as the likely dominating cause of the apparent drift. This conclusion is in line with the results of Reiners (2009), who analyze similar differential velocity drifts.

\subsection{Photospheric Rossiter-McLaughlin effect and optical photometry}

Below, we present a joint analysis of the photospheric RossiterMcLaughlin effect derived from the UVES spectra and simultaneously obtained optical photometry.

\subsubsection{Analysis of PROMT data}

The photometry was obtained using PROMPT. To reduce these data, we carried out the usual CCD data reduction steps. We identified USNO-B1.0-0913-0447626 as the most well-suited comparison star and obtained the differential light curve for CoRoT-2A along with several control light-curves using other comparison stars. To obtain the light curves, we extracted the photons from circular apertures centered on the individual stars. The local sky level was measured within an annulus surrounding the aperture. In the case of CoRoT-2A, the aperture also comprised its bona-fide physical companion, 2MASS J19270636+0122577. The resulting light curve was normalized using a linear fit to the out-of-transit points and is shown in the lower panel of Fig. 4.

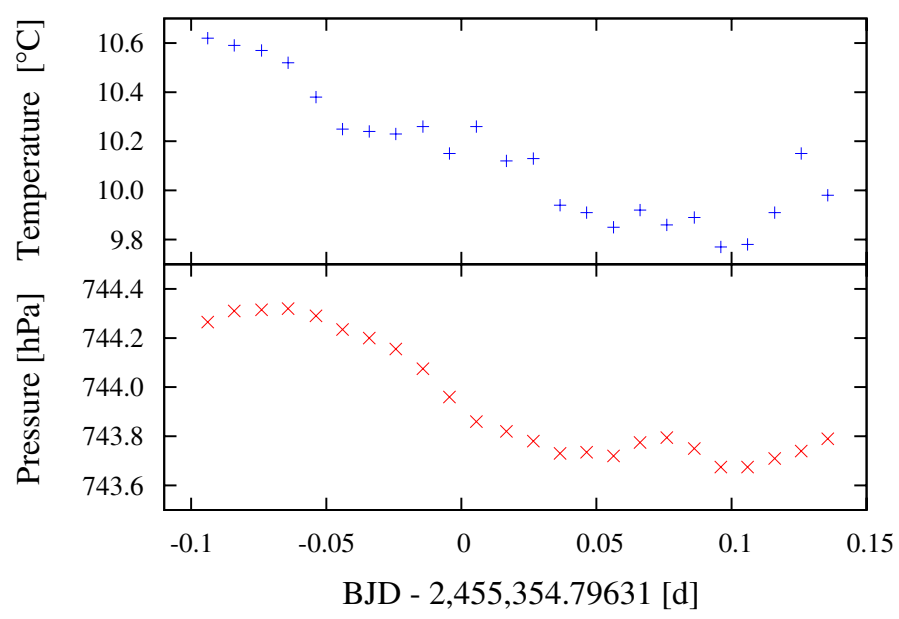

Fig. 2. Time dependence of ambient pressure and temperature during the UVES observations.

\subsubsection{Measurement of the photospheric Rossiter-McLaughlin effect}

We determined the radial-velocity shift of the stellar spectrum obtained with UVES by cross-correlating it with a synthetic template of CoRoT-2A according to the prescription by Zucker (2003). The template was calculated with the SPECTRUM stellar synthesis code (Gray \& Corbally 1994) and is based on Kurucz model atmospheres (Kurucz 1993). The stellar parameters were adopted from Schröter et al. (2011).

Before cross-correlating them, the observed spectra were continuum-normalized. To define the continuum, we manually specified an appropriate number of supporting points for a piecewise linear fit, chosen by comparing the spectra with the template. We then calculated the cross-correlation function for different parts of the spectrum that show no contamination by telluric absorption lines. While the blue parts of the spectra are most convenient from that point of view, they completely lack telluric lines that could be used to correct for the systematic instrumental drift described in Sect. 3.1. We, therefore, computed the cross-correlation in the 5700-7500 $\AA$ range and subsequently subtracted the instrumental drift inferred from the telluric lines. The result is shown in Fig. 3 along with a quadratic fit to the out-of-transit points used in the modeling.

\subsubsection{Joint modeling of photometry and Rossiter-McLaughlin effect}

In our modeling, we apply the analytical RME curves presented by Ohta et al. (2005) and the transit model developed by Pál (2008). In addition to the RME model, we take into account the orbital reflex motion of the host star using a sinusoidal curve. In our approach, we neglect the slightly nonzero $\left(e=0.0143_{-0.0076}^{+0.0077}\right)$ eccentricity determined by Gillon et al. (2010), whose influence on our model is small.

The transit model has the following parameters: the orbital period, $P$, the mid-transit time, $T_{0}$, the radius ratio, $r_{\mathrm{p}} / R_{\mathrm{s}}$, the semi-major axis in stellar radii, $a / R_{\mathrm{s}}$, the orbital inclination, $i$, the linear stellar limb-darkening coefficient, $\epsilon$, and the contribution of third light, $L_{3}$, given as a fraction of the host-star's luminosity.

The RME model of Ohta et al. (2005) encompasses, apart from the third light contribution, all parameters used in the transit model. In addition, it includes the sky-projected stellar 


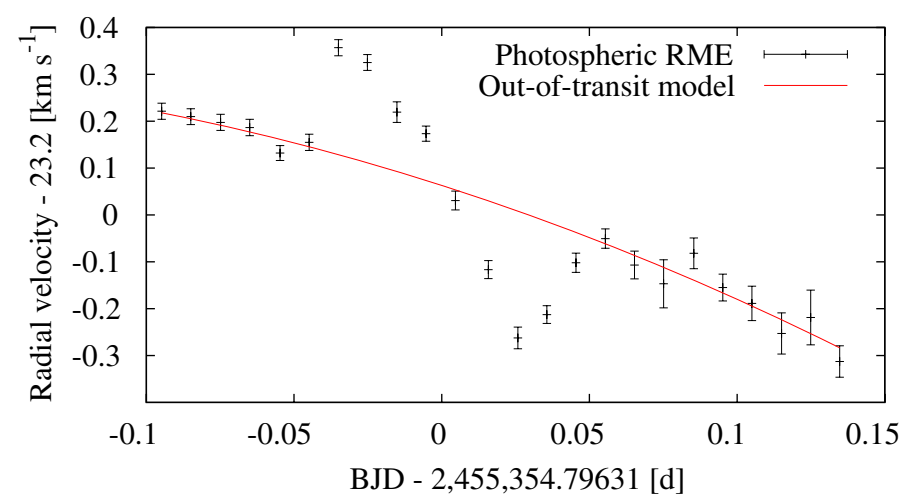

Fig. 3. The photospheric Rossiter-McLaughlin effect observed with UVES and a quadratic out-of-transit model.

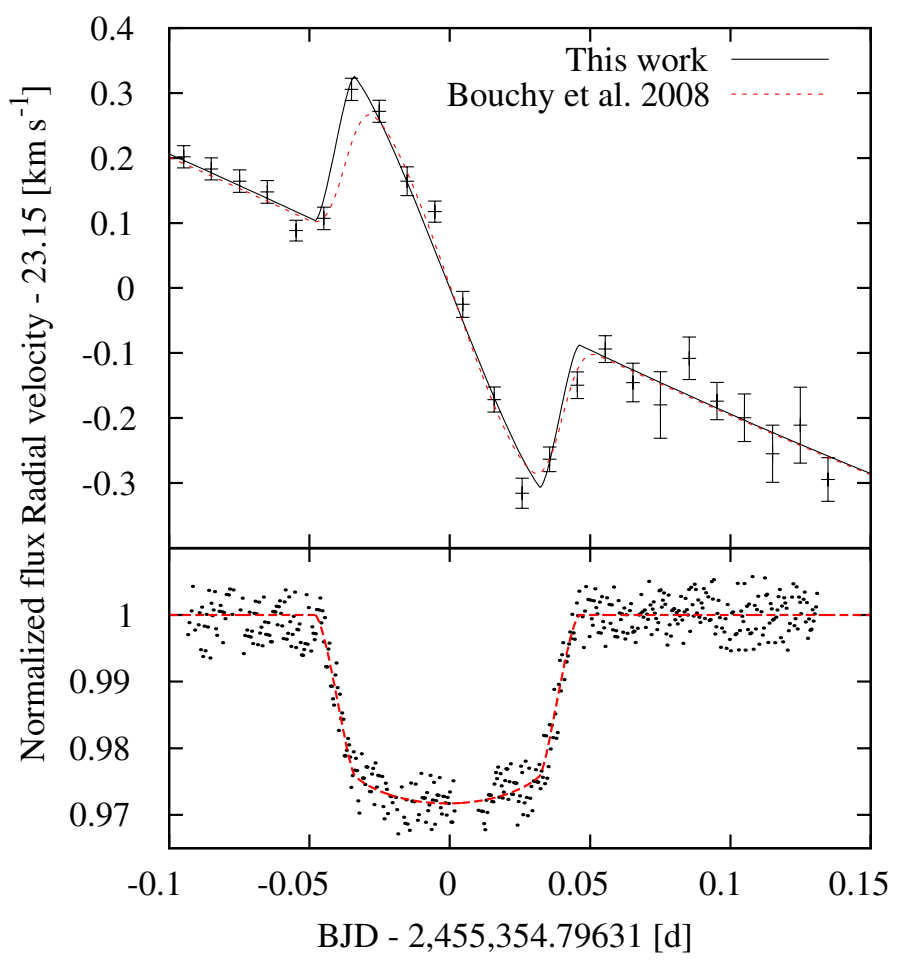

Fig. 4. Upper panel: photospheric Rossiter-McLaughlin effect of CoRoT-2A determined in the 5700-7500 Å band, our most likely model (solid), and the model given by Bouchy et al. (2008) (dashed). Lower panel: transit simultaneously observed with PROMPT and most likely model from our joint transit+RME fit.

rotational velocity, $v \sin \left(I_{\mathrm{s}}\right)$, and the sky-projected angle between stellar spin axis and planetary orbit normal, $\lambda$. Furthermore, we characterize the stellar reflex motion using a sine with RV semiamplitude, $K$, and an RV zero point, $V_{0}$. In the computation of the RV curve, we used an "overbinned" model to account for the finite $(800 \mathrm{~s})$ integration time per spectrum. In particular, each observed bin was divided into 15 model bins, which were finally averaged and compared to the observation.

In an active star such as CoRoT-2A, stellar activity could affect the measured RV curve (Albrecht et al. 2011; Bouchy et al. 2008). Additionally, systematic effects due to the observed instrumental drift may still be present. To account for RV shifts not caused by the stellar reflex motion and the RME itself, we fitted the out-of-transit points of our RV measurement using a quadratic model (see Fig. 3) and subtracted the quadratic term
Table 1. Priors and sampling results.

\begin{tabular}{lcc}
\hline \hline & Prior information & \\
Quantity $^{a}$ & Prior & Source $^{c}$ \\
\hline$v \sin \left(I_{\mathrm{s}}\right)\left[\mathrm{km} \mathrm{s}^{-1}\right]$ & $11.46 \pm 0.37$ & B08 \\
$\lambda\left[^{\circ}\right]$ & $7.1 \pm 5$ & B08 \\
$K\left[\mathrm{~km} \mathrm{~s}^{-1}\right]$ & $0.563 \pm 0.014$ & A08 \\
$i\left[^{\circ}\right]$ & $87.84 \pm 0.1$ & A08 \\
$p$ & $0.1667 \pm 0.0053$ & A08/C09 \\
$L_{3}[\%]$ & $5.6 \pm 1$ & A08 \\
$a\left[R_{\mathrm{s}}\right]$ & $6.7 \pm 0.03$ & A08 \\
$\epsilon, T_{0}, V_{0}$ & uniform & \\
$e$ & $0($ fixed $)$ & \\
\hline \multicolumn{3}{c}{ Pusterior } \\
Quantity & Value and 95\% HPD & \\
\hline$v \sin \left(I_{\mathrm{s}}\right)\left[\mathrm{km} \mathrm{s}^{-1}\right]$ & $11.95(11.4,12.53)$ & \\
$\lambda\left[^{\circ}\right]$ & $-1(-7.7,6)$ & \\
$K\left[\mathrm{~km} \mathrm{~s}{ }^{-1}\right]$ & $0.564(0.541,0.587)$ & \\
$i\left[^{\circ}\right]$ & $87.84(87.67,88)$ & \\
$p$ & $0.1639(0.1621,0.1656)$ & \\
$L_{3}[\%]$ & $5.2(3.2,7.1)$ & \\
$a\left[R_{\mathrm{s}}\right]$ & $6.73(6.69,6.76)$ & \\
$\epsilon$ & $0.33(0.28,0.37)$ & \\
$T_{0}[\mathrm{~s}]$ & $-76.7(-88.2,-65.3)$ & \\
$V_{0}[\mathrm{~m} / \mathrm{s}]$ & $63.3(52.9,72.6)$ & \\
\hline
\end{tabular}

Notes. ${ }^{(a)}$ We use the nomenclature of Ohta et al. (2005); in addition, $K$ is the radial velocity semi-amplitude, $V_{0}$ the $\mathrm{RV}$ offset, and $L_{3}$ the third light contribution. ${ }^{(b)}$ In parentheses, the $95 \%$ HPD credibility intervals are given. ${ }^{(c)}$ A08 = Alonso et al. (2008), B08 = Bouchy et al. (2008), C09 = Czesla et al. (2009).

(4.08 $\mathrm{km} \mathrm{d}^{-2}$ ). The resulting RV curve is shown in the top panel of Fig. 4 and was used in the modeling.

The parameters in question had already been measured in previous works. To take the existing information into account in our modeling, we specified Gaussian priors based on previous results for the parameters: $v \sin \left(I_{\mathrm{s}}\right), \lambda, K, V_{0}, r_{\mathrm{p}} / R_{\mathrm{s}}, L_{3}, a$, and $i$. For the radius ratio, $r_{\mathrm{p}} / R_{\mathrm{s}}$, we used the difference between the values derived by Alonso et al. (2008) and Czesla et al. (2009) as the width of the prior. For all other parameters, except for the eccentricity, which was neglected, we used uniform priors covering the full physically reasonable space. The information on parameters and priors is summarized in Table 1.

We proceeded by sampling from the posterior probability distribution using a Markov-chain Monte Carlo (MCMC) approach. In Fig. 4, we show the most likely model given the data and the prior information. The expectation values for the parameters and $95 \%$ credibility intervals derived from the posterior are given in Table 1. We note that the prior information has a noticeable influence on the posterior, especially that on the stellar rotation velocity, $v \sin \left(I_{\mathrm{s}}\right)$. If we neglect this prior information by using a broad uniform prior, the posterior yields $v \sin \left(I_{\mathrm{S}}\right)=12.9(11.9,13.9) \mathrm{km} \mathrm{s}^{-1}$, increasing the amplitude of the RME model.

Our analysis shows that CoRoT-2A's companion, 2MASS J19270636+0122577, provides $5.2 \%$ of the total flux in the PROMPT band, which is comparable to the number of $5.6 \%$ derived by Alonso et al. (2008) in the CoRoT band. The observed transit center, $T_{0}$, shows a slight displacement of $-76.7 \mathrm{~s}$ if compared to the ephemerides given by Alonso et al. (2008). 
The numbers are, however, compatible considering that the $1 \sigma$ uncertainty in the Alonso et al. ephemerides amounts to $95 \mathrm{~s}$ at the given epoch, which covers our value. Using the reference point, $T_{0, \mathrm{~A}}$, given by Alonso et al. and our transit center, $T_{0}$, we can slightly improve the estimate of the planet's orbital period using the equation

$$
\begin{aligned}
P_{\mathrm{o}} & =\frac{(2455354.796312 \mathrm{~d}-76.7 \mathrm{~s})-T_{0, \mathrm{~A}}}{641} \\
& =1.74299502 \pm 0.000001 \mathrm{~d} .
\end{aligned}
$$

We caution that large starspots may affect the transit timing, but as our period estimate lies within the error given by Alonso et al. (2008), their effect is unlikely to be larger than the observed offset of $-77 \mathrm{~s}$. Whether the small deviation is due to starspots, cannot be decided on the basis of the data at hand.

In the upper panel of Fig. 4, we show our data points (see also Table A.1), our best-fit RME model, and the model derived by Bouchy et al. (2008) (their "combined MCMC fit" shifted to our RV offset); in Table 1, we list the associated parameter values and the $95 \%$ highest probability density (HPD) credibility intervals derived using MCMC sampling.

The RME observed with UVES shows a slightly larger amplitude than reported by Bouchy et al. (2008), which causes the larger $v \sin \left(I_{\mathrm{S}}\right)$ value derived from our data. While the derived linear limb-darkening coefficient, $\epsilon$, of 0.3 appears to be low compared to the number of 0.66 reported by Claret (2004) for a star like CoRoT-2A in the $r^{\prime}$ band, it is in better agreement with the number of $0.41 \pm 0.03$ given by Alonso et al. (2008), neglecting their small (0.06) quadratic term.

Although the amplitude of the RV shift produced by the orbital motion of the planet, $K$, cannot be reliably determined given that our data cover only about $10 \%$ of the orbital period, we find our number of $564(541,587) \mathrm{m} \mathrm{s}^{-1}$ to be in good agreement with the value of $563 \pm 14 \mathrm{~m} \mathrm{~s}^{-1}$ derived by Alonso et al. (2008) using the full orbit.

CoRoT-2A is an active star, which had a substantial spot coverage during the half-year long CoRoT observation (Alonso et al. 2008; Czesla et al. 2009; Huber et al. 2010). It is likely that spots were also present during the transit under consideration. Parameter uncertainties imposed by stellar activity on the order of a few percent have been found for CoRoT-2A, e.g., by Czesla et al. (2009), and we expect to find a similar effect in our current measurements.

Our photometry suggests that the planet-to-star radius ratio is about $2 \%$ smaller than observed by Alonso et al. (2008). Such a decrease may be caused by a corresponding decrease in the total spot coverage of the star, which would make the planet appear smaller. A smaller planet could also account for the larger model value of the stellar rotation velocity, which would then counterbalance the less pronounced RME effect produced by a smaller planet. Alternatively, the spot coverage on the eclipsed section of the star may be larger, thus, making the transit appear less deep (Czesla et al. 2009). We refrain from pointing out individual spot-crossing events in our photometry, because the data do not allow a unique identification.

Strong stellar activity as observed on CoRoT-2A can mask the true values of the physical parameters, leading to differences between individual measurements. To quantify this activity scatter, a larger sample of measurements, allowing a statistical analysis, would be needed.

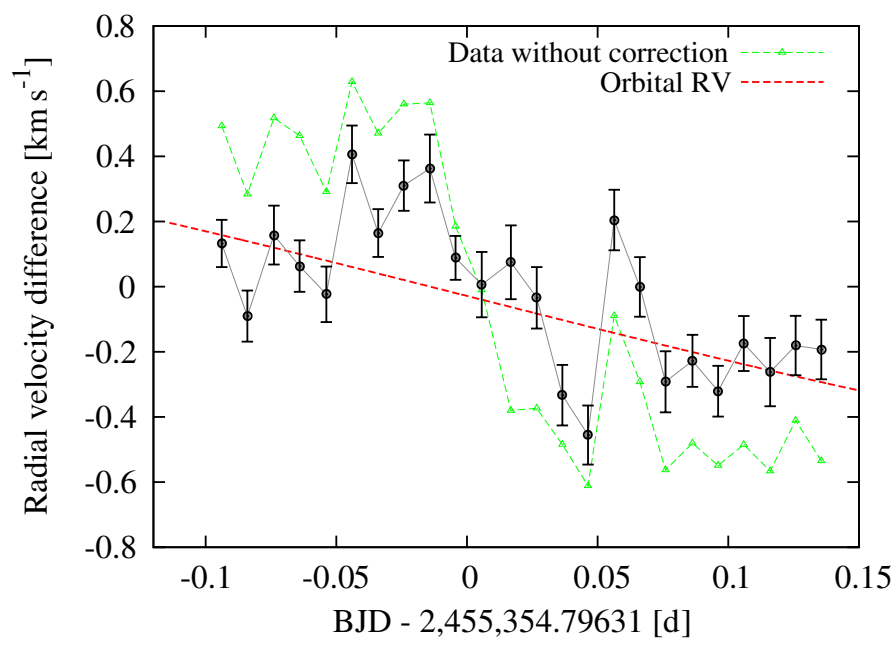

Fig. 5. Chromospheric Rossiter-McLaughlin effect: radial velocity shift of the $\mathrm{Ca}$ II $\mathrm{H}$ and $\mathrm{K}$ lines. Circular data points: $\mathrm{RV}$ corrected for telluric drift; triangles: uncorrected RV shift; and dashed line: orbital radial velocity model.

\subsection{The chromospheric Rossiter-McLaughlin effect}

As the planet eclipses the chromosphere during the transit, the RME should also be observable in chromospheric emission lines. Among the chromospheric features in CoRoT-2A's spectrum, we find that only the cores of the $\mathrm{Ca}$ II $\mathrm{H}$ and $\mathrm{K}$ lines are usable for our analysis. These lines are both strong enough and sufficiently uncontaminated by photospheric emission. A detailed analysis of the chromospheric features in $\mathrm{H} \alpha, \mathrm{H} \beta$, the $\mathrm{Na}$ I lines, or the $\mathrm{Ca}$ infrared triplet is impeded by a comparably large photospheric background, which is small in the Ca II $\mathrm{H}$ and $\mathrm{K}$ emission-line cores (see Fig. 7, or Fig. 4 in Schröter et al. 2011).

In the red, we used the telluric lines as standards to improve our wavelength calibration (cf., Sect. 3.1). Because this procedure remains impossible in the blue owing to the lack of appropriate telluric lines, we adopted another approach to obtain a correction for the instrumental drift: We derived the photospheric RME in the blue part of the spectrum using a synthetic spectrum and a line list obtained from VALD (Piskunov et al. 1995) to determine the wavelength of 38 unblended stellar absorption lines between 3800 and $4300 \AA$. Our previously calculated red RME model was then subtracted and the residual signal was attributed to the drift. While we used the thus derived correction to model the chromospheric RME, we emphasize that the detection of the effect is independent of this correction.

To estimate the apparent RV shift of the $\mathrm{Ca}$ II $\mathrm{H}$ and $\mathrm{K}$ line cores, we determined their barycenters using small $0.5 \AA$-wide segments centered around the cores' nominal positions (cf., Fig. 7). As both should be similarly affected, we averaged the results yielding a mean $\mathrm{RV}$ shift in the $\mathrm{Ca}$ II $\mathrm{H}$ and $\mathrm{K}$ emission line cores.

In an alternative approach, we approximated the $\mathrm{Ca}$ II $\mathrm{H}$ and $\mathrm{K}$ emission-line cores with Gaussians and used MCMC sampling to explore the posterior probability distribution. The measurement errors were assumed to obey a normal distribution and were derived by comparing the observed spectrum to an appropriate synthetic template. Both the barycenter and the Gaussian fit approach yielded comparable results. The resulting RV shifts are listed in Table A.1, and the outcome of the MCMC analysis is shown in Fig. 5, which shows an RV curve with a signature strongly reminiscent of the photospheric RME; the errors 
correspond to $68 \%$ credibility intervals. We found that the center of the Gaussian is not correlated with any other parameter and concluded that the estimate is insensitive to the shape of the emission-line core.

To check the significance of the detection, we tested the null hypothesis that the $\mathrm{RV}$ variation in the $\mathrm{Ca}$ II $\mathrm{H}$ and $\mathrm{K}$ cores is described by the orbital motion of the planet alone. Applying $\chi^{2}$ statistics, the null hypothesis can be rejected with a $p$-value of $10^{-10}$, indicating that our data include an additional contribution to the RV variation, which we attribute to the chromospheric RME.

To exclude the possibility that we merely detected the RME present in the residual photospheric flux underneath the Ca II $\mathrm{H}$ and $\mathrm{K}$ emission-line cores, we used simulated spectra. First, we obtained a synthetic spectrum of the $\mathrm{Ca}$ II $\mathrm{H}$ and $\mathrm{K}$ line region without emission-line cores, shifted it according to the observed photospheric RME, and applied noise to simulate the observation. We then used the same barycenter method applied to the UVES spectra and found that no RV shift can be detected that can be distinguished from noise. This is a consequence of the broad $\mathrm{Ca}$ II $\mathrm{H}$ and $\mathrm{K}$ line wings. Second, we added the emissionline cores. Our simulations show that both the RV signal resulting from the barycenter method and the Gaussian fit to the cores are dominated by the shift in the cores and virtually unaffected by the underlying photospheric flux. We, therefore, conclude that the observed additional RV shift is not a relic of the photospheric RME, but is, indeed, related to the chromospheric emission.

\subsubsection{Interpreting the radial velocity signature}

The similarity of the chromospheric RV signature (see Fig. 5) and the photospheric RME suggests that the surface of CoRoT-2A is covered by active regions - at least the fraction eclipsed by the planet. The chromospheric and photospheric RME can, however, be described by the same model with different parameter values accounting for the physical conditions in the chromosphere and the photosphere. In particular, the wavelength-dependent center-to-limb variation and a, potentially, wavelength-dependent radius ratio must be taken into account (Vidal-Madjar et al. 2003; Dreizler et al. 2009).

In a first attempt to model the chromospheric RME, we considered only the RV offset, $V_{0}^{\mathrm{c}}$, and the center-to-limb variation, i.e., the limb-darkening coefficient, $\epsilon$, as free parameters. The remaining model parameters remained fixed at the photospheric values given in Table 1 . We, again, imposed uniform priors on all parameters and used MCMC sampling to explore the posterior probability distribution. The optimal solution found after $10^{6}$ iterations is shown in Fig. 6. The fit describes the data qualitatively, but, formally, the quality remains poor with a $\chi^{2}$-value of 76.3 for 22 degrees of freedom.

In a second fitting attempt, we allowed for an extended chromosphere, i.e., a larger radius of the star, by introducing a scaling factor. Using a uniform prior for the scaling, we repeated the MCMC sampling and found that the data are reproduced best if the chromospheric radius is a factor of $1.16(1.1,1.23)$ larger than the photospheric radius, $R_{\mathrm{ph}}$. Formally, the fit is improved yielding a $\chi^{2}$-value of 58.8 with 21 degrees of freedom for the lowest deviance solution.

Clearly, the $95 \%$ highest probability density (HPD) interval favors a larger chromospheric radius (see Table 2). To solidify

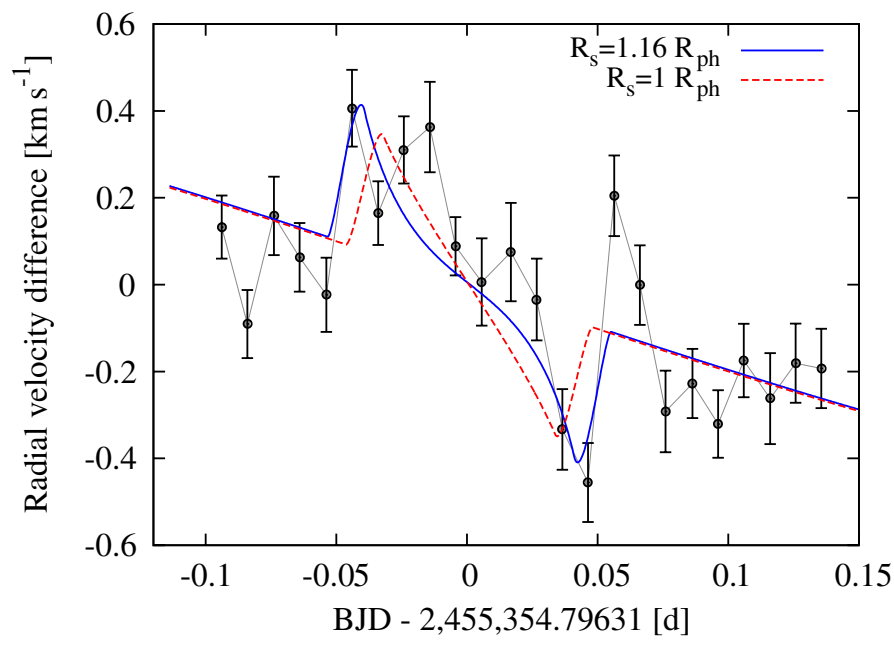

Fig. 6. Chromospheric Rossiter-McLaughlin effect: data points (filled black circles), best-fit model with radii fixed at photospheric values (dashed red), and best-fit model with free stellar radius (solid blue).

Table 2. Results of the modeling of the chromospheric RossiterMcLaughlin effect.

\begin{tabular}{lll}
\hline \hline Quantity & Value $^{a}$ & $95 \% \mathrm{HPD}$ \\
\hline$\epsilon$ & -0.4 & {$\left[-16.5,1.0^{b}\right]$} \\
$V_{0}^{\mathrm{c}}\left[\mathrm{km} \mathrm{s}^{-1}\right]$ & 21.953 & {$[21.918,21.987]$} \\
\hline$\epsilon$ & -4.4 & {$\left[-20.0^{b},-0.6\right]$} \\
$V_{0}^{\mathrm{c}}\left[\mathrm{km} \mathrm{s}^{-1}\right]$ & 21.957 & {$[21.922,21.992]$} \\
$R_{\mathrm{S}}\left[R_{\text {photo }}\right]$ & 1.16 & {$[1.10,1.23]$} \\
\hline
\end{tabular}

Notes. ${ }^{(a)}$ We report the value pertaining to the lowest deviance solution after $10^{6}$ iterations. ${ }^{(b)}$ This limit of the credibility interval is determined by the finite range of our uniform prior.

the significance of the result, we used an $F$-test to compare the vying models. We calculated the estimator

$\hat{F}=\frac{\left(\chi_{a}^{2}-\chi_{b}^{2}\right) /\left(v_{a}-v_{b}\right)}{\chi_{b}^{2} / v_{b}}$

and compared its value to an $F$-distribution with $v_{a}-v_{b}$ and $v_{b}$ degrees of freedom (e.g., Rawlings et al. 1998). We found that the improvement is significant at the $98 \%$ confidence level.

In an alternative approach, we used the method described by Newton \& Raftery (1994, see their Sect. 7, Eq. (13)) to estimate the marginal likelihood using the harmonic mean computed from the posterior sample generated by the Markov chain. Given the marginal likelihoods, we computed the Bayes factor, $B$, and obtained $\log _{10}(B)=3.5$. In the scale introduced by Jeffreys (see, e.g., Kass \& Raftery 1995, and references), this yields "decisive" evidence in favor of the model that proposes a larger chromospheric radius for CoRoT-2A. We conclude that this model provides a significantly better description of the data.

We note that a model in which the planetary radius is enlarged to account for the prolonged transit is not supported by our data. To explain the longer transit, the planetary radius would have to be larger by about a factor of two, which would drastically increase the amplitude of the RME as a whole and is inconsistent with the data.

Taking the stellar radius into account, the relative enlargement of $16 \%$ can be converted into an absolute "chromospheric" scale of $100000 \mathrm{~km}$. For the Sun, Beck \& Rezaei (2011) observed $\mathrm{Ca} \mathrm{H}$ and $\mathrm{K}$ emission up to $5000 \mathrm{~km}$ above the solar 
limb. The largest solar prominences such as the "Grand Daddy Prominence" reach projected heights of $112000 \mathrm{~km}$ as measured in $\mathrm{H} \alpha$ emission (Pettit 1946). Reverting to the solar analog, we speculate that CoRoT-2A is covered with structures reminiscent of the Grand Daddy Prominence in size. Whether these structures are stable cannot be decided on the basis of the data, although, in our measurement, the chromospheric RME appears to be symmetric, which indicates the presence of extended structures on both sides of the star. We interpret this as evidence of a stable configuration or at least a high filling factor of active regions creating large chromospheric structures.

We found that $\epsilon$, which parametrizes the chromospheric center-to-limb variation, appears to be negative. This indicates that the chromosphere is limb-brightened. At some point, the limb-brightened RME model effectively becomes a "ring model", which is relatively indifferent to further changes in $\epsilon$. As our data do not rule out this region, the exact level of chromospheric limb-brightening cannot currently be determined.

As shown in Fig. 6, some data points are incompatible with either RME model. This may be due to the following reasons: statistics and systematics, chromospheric inhomogeneities, and intrinsic variability.

During the transit, the RME curve could be affected by plage-crossing events. We checked that features with RV amplitudes of $\approx 150 \mathrm{~m} \mathrm{~s}^{-1}$ can be reproduced assuming a plage region with a size similar to that of the planetary disk and twice the photospheric brightness (cf., Sect. 3.3.2). Additionally, the chromospheric emission may show intrinsic variability unrelated to the transit. However, we argue that neither intrinsic variability nor systematics due to the wavelength calibration are likely to mimic the prolonged transit signature observed in $\mathrm{Ca}$ II $\mathrm{H}$ and $\mathrm{K}$. Nevertheless, such an effect cannot ultimately be excluded.

\subsubsection{Distribution of chromospheric and photospheric emission}

The detection of the RME in the $\mathrm{Ca}$ II $\mathrm{H}$ and $\mathrm{K}$ lines shows that a significant fraction of the chromospheric emission regions must be eclipsed during the entire transit. Any differences in the distribution of chromospheric and photospheric surface brightnesses should be reflected by a variable equivalent width (EW) of the $\mathrm{Ca}$ II $\mathrm{H}$ and $\mathrm{K}$ emission-line cores during the transit, because chromospheric emission would be measured relative to the photospheric continuum. To obtain an estimate of the EW, we summed the signal in $1.4 \AA$ wide bands centered on the $\mathrm{Ca}$ II $\mathrm{H}$ and $\mathrm{K}$ emission-line cores (see Fig. 7) and compared it to the signal obtained in the two photospheric bands in the regions 3981-3996 $\AA$ and 3861-3909 A. The choice of these bands is arbitrary, but we verified that our results depend only weakly on this choice. The resulting ratio, $R$, is a measure of the quantity

$R(t)=\frac{\left\langle b_{\mathrm{vis}}\left(\lambda_{\mathrm{Ca}}\right)\right\rangle(t)}{\left\langle b_{\mathrm{vis}}\left(\lambda_{\mathrm{Ph}}\right)\right\rangle(t)}$,

where $\left\langle b_{\mathrm{vis}}\left(\lambda_{\mathrm{Ca}, \mathrm{Ph}}\right)\right\rangle$ is the mean surface brightnesses of the visible fraction of the star in $\mathrm{Ca}$ II $\mathrm{H}$ and $\mathrm{K}$ and the photosphere.

Figure 8 shows the outcome. No variation in the observed EW is detectable within the limits of our uncertainties. The ratio of $\mathrm{Ca}$ II $\mathrm{H}$ and $\mathrm{K}$ signal to the continuum signal in the aforementioned bands amounts to $R=(2.31 \pm 0.028) \times 10^{-2}$. The given error corresponds to the sample standard deviation. It does not differ for in- and out-of-transit points and is attributed to a combination of measurement errors and intrinsic variability.

If the photospheric and chromospheric emission were equally distributed on the visible surface of CoRoT-2A, the EW
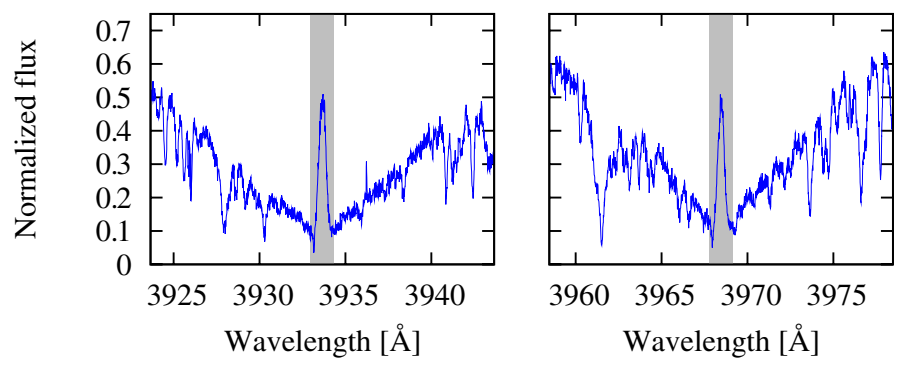

Fig. 7. CoRoT-2A's Ca II H and $\mathrm{K}$ lines. Gray intervals indicate the bands used to measure the flux in the emission-line cores (see text for details).

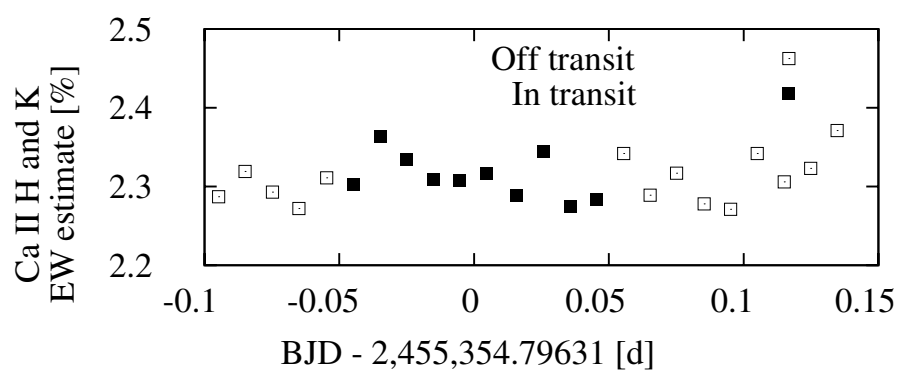

Fig. 8. Ratio of integrated fluxes in both the $\mathrm{Ca}$ II $\mathrm{H}$ and $\mathrm{K}$ emission-line cores (see Fig. 7) to those in the comparison bands (see text).

of the $\mathrm{Ca}$ II $\mathrm{H}$ and $\mathrm{K}$ cores and, therefore, $R$ would remain constant during transit, because the occultation has the same effect on both photospheric and chromospheric emission. A change in $R$ indicates a deviation from the equal distribution on the currently occulted section of the stellar disk. If, for example, an active region is occulted, more chromospheric than photospheric emission should be blocked, leading to a decrease in $R$.

In the following, we quantify the relation between surface brightness distribution and $R$. The brightness, $B(\lambda)$, of the entire stellar disk at any wavelength, $\lambda$, can be written as

$B(\lambda)=f\left\langle b_{\mathrm{occ}}(\lambda)\right\rangle+F\left\langle b_{\mathrm{vis}}(\lambda)\right\rangle$

where $f$ is the occulted area of the stellar disk and $F$ the visible area. For the special case of $\left\langle b_{\text {occ }}\right\rangle=\left\langle b_{\text {vis }}\right\rangle=b_{0}$, we obviously obtain $B=(f+F) b_{0}$. Starting from exactly this situation, we introduce a bright chromospheric plage region with a local surface brightness, $\left\langle b_{\mathrm{occ}}^{\prime}\left(\lambda_{\mathrm{Ca}}\right)\right\rangle$, which is a factor of $\alpha$ higher than the mean surface brightness. Hence, we write $\left\langle b_{\text {occ }}^{\prime}\left(\lambda_{\mathrm{Ca}}\right)\right\rangle=$ $\alpha b_{0}\left(\lambda_{\mathrm{Ca}}\right)$.

The conservation of total brightness demands that a local increase in the surface brightness across the eclipsed section of the star must be balanced by an adjustment of the mean brightness of the visible fraction, $\left\langle b_{\mathrm{vis}}\left(\lambda_{\mathrm{Ca}}\right)\right\rangle$, which we parametrize by $\beta$ so that $\left\langle b_{\text {vis }}^{\prime}\left(\lambda_{\mathrm{Ca}}\right)\right\rangle=\beta b_{0}$.

Assuming that the photospheric surface brightness remains unaffected, the ratio $R$ is proportional to $\beta$ (see Eq. (3)). Starting from $B=f \alpha b_{0}+F \beta b_{0}$, we obtain the relation

$\beta=1+\frac{f}{F}(1-\alpha)$

In the case of CoRoT-2A, the factor $f / F$ amounts to $\approx 0.03$. Thus, if a plage region with a local $\mathrm{Ca}$ II $\mathrm{H}$ and $\mathrm{K}$ surface 
brightness that is a factor of $\alpha=15$ higher than the mean brightness were eclipsed, the observed EW would be a factor of 2 lower. The relative stability of $R$, which varies by only about $3 \%$, suggests that the surface brightness of the Ca II $\mathrm{H}$ and $\mathrm{K}$ emission does not strongly deviate from that of the photosphere. According to our result, the photospheric and chromospheric surface brightnesses are the same to within a factor of about 2 .

An analysis of the chromospheric emission in the Ca II infrared triplet, which is visible as a substantial filling-in of the line cores, yielded compatible but less conclusive results due to the much larger photospheric contribution.

\subsection{Searching for planetary Na I absorption}

During a primary eclipse, stellar light passes through the planetary atmosphere, potentially giving rise to planetary absorption features. These features have, indeed, been detected in several systems using the Hubble Space Telescope and more recently ground-based data (e.g., Charbonneau et al. 2002; Redfield et al. 2008). According to Seager \& Sasselov (2000), the most prominent planetary absorption features in the optical should be caused by neutral sodium.

To search for planetary sodium absorption features in our spectra, we combined them to yield in- and out-of-transit templates with a $\mathrm{S} / \mathrm{N}$ ratio of 90 and 110 around $\mathrm{NaI}$, respectively. As discussed by Schröter et al. (2011), the sodium doublet is affected by strong blue-shifted interstellar absorption. Additionally, the line core is contaminated by residual sky emission. In the course of our observations, the strength of the sky contribution increased monotonically following the airmass. We found that a correction for the sky emission is problematic, because the sky spectrum shifts in wavelength relative to the stellar spectrum producing spurious absorption in the in-transit template. The quality of our combined template spectra does not allow us to subtract a heuristic model of the sky emission without introducing additional residuals. Therefore, we found it impossible to deduce a reasonable upper limit to the planetary sodium absorption.

\section{Discussion and conclusion}

We have presented our analysis of 24 high-resolution UVES spectra of CoRoT-2A and simultaneously obtained photometry, observed during a planetary transit. We have reported on the - to the best of our knowledge - first detection of a prolonged $\mathrm{RME}$ in the chromospheric $\mathrm{Ca}$ II $\mathrm{H}$ and $\mathrm{K}$ emission-line cores. Furthermore, we have presented a joint analysis of the photospheric RME and the transit photometry.

During our analysis, we found that the wavelength calibration of UVES undergoes a substantial drift, which we corrected using telluric standards. While the modeling of the RME depends on the details of this correction, neither the detection of the photospheric nor the chromospheric RME does.

Our modeling yielded an improved estimate of the orbital period of the planet and shows that our data favor an RME amplitude slightly larger than that reported by Bouchy et al. (2008) using HARPS and SOPHIE data. This results in a larger estimate of the projected rotational velocity, $v \sin \left(I_{\mathrm{S}}\right)$, of the star. This difference potentially reflects a change in the total stellar spot coverage between the observations. As Alonso et al. (2008), we derived a weak limb-darkening relative to the theoretical numbers given by Claret (2004). We speculate that a larger contribution from plage regions and, therefore, the observed activity, may be responsible for this. While the planet clearly passes across the stellar disk, our search for planetary absorption features in sodium remained inconclusive, owing to the lack of signal and contaminating sky emission.

The presence of the chromospheric RME indicated that chromospheric emission was eclipsed during every phase of the visual transit - and beyond. We present evidence that the transit observed in $\mathrm{Ca}$ II $\mathrm{H}$ and $\mathrm{K}$ lasts about $15 \%$ longer than its visual counterpart. Our modeling shows that the data are most closely reproduced by increasing the size of the stellar chromosphere. In contrast, boosting the planetary radius by a factor of two, as would be needed to explain the longer transit, is incompatible with the data. The observed chromospheric scale of $100000 \mathrm{~km}$ is compatible with large solar structures such as the "Grand Daddy Prominence". Our observations indicate that such structures could cover a substantial fraction of the surface of CoRoT-2A and other very active stars.

The analysis of the chromospheric RME favors chromospheric limb-brightening. However, in a subsequent analysis of the EW of the Ca II $\mathrm{H}$ and $\mathrm{K}$ emission-line cores, we found no differences between the surface brightness distributions of chromospheric and photospheric emission to within the uncertainties of our data.

Besides the scale height of the chromosphere, the analysis of the chromospheric RME allows to explore the structure of the stellar chromosphere covered by the planetary disk, i.e., the center-to-limb variation and inhomogeneities due to active regions. Indeed, the asymmetry of the chromospheric RME curve (see Fig. 6; $0<$ time $<0.05$ d) may be a consequence of inhomogeneities in the chromospheric surface-brightness distribution. However, the sparse phase coverage, the uncertainties imposed by the instrumental RV drift, and the likely presence of intrinsic variability do not allow to safely attribute these variations to surface features. Nonetheless, both points could be addressed by dedicated observations with today's instrumentation. In particular, what is needed to verify our results is higher temporal resolution, spectra of equivalent or higher $\mathrm{S} / \mathrm{N}$, and improved RV stability - hence, a brighter target.

To increase the amount of chromospheric signal, future observations could include other emission-line cores in the UV region such as the $\mathrm{Mg}$ II $\mathrm{H}$ and $\mathrm{K}$ lines at $\approx 2800 \AA$, which are not covered by the present data. As for $\mathrm{Ca}$ II $\mathrm{H}$ and $\mathrm{K}$, these lines have weak photospheric contributions, but are, unfortunately, impossible to observe from the ground.

As an alternative to the chromospheric RME, the chromospheric center-to-limb variation and inhomogeneities could also be studied using narrow-band transit-photometry centered on chromospheric emission lines (Assef et al. 2009; Schlawin et al. 2010). In contrast to the RME, however, this method relies on a precise photometric calibration (Snellen 2004), which is difficult to achieve especially in ground-based observations.

Our analysis demonstrates the power of the RossiterMcLaughlin effect for the exploration of the stellar atmosphere and clearly underlines the need for dedicated observations.

Acknowledgements. We thank the anonymous referee for thoughtful comments. This work makes use of observational data obtained with UVES at the ESO Very Large Telescope, Paranal, Chile. S.C., C.v.E., and K.F.H. are supported in the framework of the DFG-funded Research Training Group "Extrasolar Planets and their Host Stars" (DFG 1351/1). and U.W. acknowledge support from the DLR under grant 50OR0105. S.S. acknowledges support from the DLR under grant 50OR0703. PROMPT observations were made possible by the Robert Martin Ayers Science Fund. 


\section{Appendix A: Details of observations and data analysis}

Table A.1. Details of data and data analysis.

\begin{tabular}{|c|c|c|c|c|c|c|c|c|c|}
\hline No. & $\begin{array}{c}\text { HJD } \\
-2400000 \mathrm{~d}\end{array}$ & $\overline{\mathrm{IS}^{a}}$ & Airmass & $\begin{array}{c}\text { Seeing } \\
{\left[{ }^{\prime \prime}\right]}\end{array}$ & $\begin{array}{c}\text { Pressure } \\
{[\mathrm{hPa}]}\end{array}$ & $\begin{array}{l}\text { Temp. } \\
{\left[{ }^{\circ} \mathrm{C}\right]}\end{array}$ & $T^{b}$ & $\begin{array}{c}\mathrm{RV} \\
{\left[\mathrm{km} \mathrm{s}^{-1}\right]}\end{array}$ & $\begin{array}{c}\Delta \mathrm{RV} \mathrm{Ca} \mathrm{II} \\
{\left[\mathrm{km} \mathrm{s}^{-1}\right]}\end{array}$ \\
\hline 1 & 55354.701526 & $\mathrm{n}$ & 1.377 & 0.94 & 744.27 & 10.62 & $\mathrm{n}$ & $23.424 \pm 0.017$ & $0.16 \pm 0.07$ \\
\hline 2 & 55354.711337 & $\mathrm{n}$ & 1.319 & 1.07 & 744.31 & 10.59 & $\mathrm{n}$ & $23.412 \pm 0.017$ & $-0.06 \pm 0.08$ \\
\hline 3 & 55354.721503 & $\mathrm{n}$ & 1.270 & 0.89 & 744.32 & 10.57 & $\mathrm{n}$ & $23.400 \pm 0.017$ & $0.19 \pm 0.09$ \\
\hline 4 & 55354.731315 & $\mathrm{n}$ & 1.231 & 1.08 & 744.32 & 10.52 & $\mathrm{n}$ & $23.389 \pm 0.017$ & $0.09 \pm 0.08$ \\
\hline 5 & 55354.741612 & $\mathrm{n}$ & 1.196 & 0.95 & 744.29 & 10.38 & $\mathrm{n}$ & $23.335 \pm 0.016$ & $0.01 \pm 0.09$ \\
\hline 6 & 55354.751424 & $\mathrm{n}$ & 1.170 & 1.03 & 744.25 & 10.26 & $\mathrm{y}$ & $23.357 \pm 0.017$ & $0.44 \pm 0.09$ \\
\hline 7 & 55354.761459 & $\mathrm{n}$ & 1.149 & 1.07 & 744.19 & 10.24 & $\mathrm{y}$ & $23.559 \pm 0.017$ & $0.20 \pm 0.07$ \\
\hline 8 & 55354.771271 & $\mathrm{n}$ & 1.133 & 1.12 & 744.16 & 10.23 & $\mathrm{y}$ & $23.528 \pm 0.017$ & $0.34 \pm 0.08$ \\
\hline 9 & 55354.781191 & $\mathrm{n}$ & 1.122 & 1.36 & 744.08 & 10.26 & $\mathrm{y}$ & $23.422 \pm 0.022$ & $0.41 \pm 0.10$ \\
\hline 10 & 55354.791007 & $\mathrm{n}$ & 1.115 & 1.31 & 743.96 & 10.20 & $\mathrm{y}$ & $23.376 \pm 0.016$ & $0.12 \pm 0.07$ \\
\hline 11 & 55354.801043 & $\mathrm{n}$ & 1.113 & 1.15 & 743.89 & 10.26 & $\mathrm{y}$ & $23.233 \pm 0.020$ & $0.03 \pm 0.10$ \\
\hline 12 & 55354.812237 & $\mathrm{y}$ & 1.116 & 1.45 & 743.80 & 10.12 & $\mathrm{y}$ & $23.086 \pm 0.019$ & $0.08 \pm 0.11$ \\
\hline 13 & 55354.822116 & $\mathrm{y}$ & 1.124 & 1.29 & 743.78 & 10.13 & $\mathrm{y}$ & $22.940 \pm 0.023$ & $-0.04 \pm 0.09$ \\
\hline 14 & 55354.831931 & $\mathrm{y}$ & 1.135 & 0.98 & 743.73 & 9.91 & $\mathrm{y}$ & $22.990 \pm 0.019$ & $-0.32 \pm 0.09$ \\
\hline 15 & 55354.841743 & $\mathrm{y}$ & 1.152 & 0.98 & 743.74 & 9.91 & $\mathrm{y}$ & $23.100 \pm 0.020$ & $-0.44 \pm 0.09$ \\
\hline 16 & 55354.851776 & $\mathrm{y}$ & 1.174 & 0.95 & 743.70 & 9.85 & $\mathrm{n}$ & $23.152 \pm 0.021$ & $0.23 \pm 0.09$ \\
\hline 17 & 55354.861589 & $\mathrm{y}$ & 1.201 & 0.92 & 743.78 & 9.92 & $\mathrm{n}$ & $23.096 \pm 0.030$ & $0.03 \pm 0.09$ \\
\hline 18 & 55354.871404 & $\mathrm{y}$ & 1.235 & 0.83 & 743.80 & 9.86 & $\mathrm{n}$ & $23.056 \pm 0.051$ & $-0.26 \pm 0.09$ \\
\hline 19 & 55354.881691 & $\mathrm{y}$ & 1.277 & 0.85 & 743.75 & 9.89 & $\mathrm{n}$ & $23.121 \pm 0.033$ & $-0.20 \pm 0.08$ \\
\hline 20 & 55354.891506 & $\mathrm{y}$ & 1.326 & 0.83 & 743.68 & 9.77 & $\mathrm{n}$ & $23.048 \pm 0.029$ & $-0.29 \pm 0.08$ \\
\hline 21 & 55354.901320 & $\mathrm{y}$ & 1.385 & 0.71 & 743.68 & 9.78 & $\mathrm{n}$ & $23.014 \pm 0.037$ & $-0.15 \pm 0.09$ \\
\hline 22 & 55354.911400 & $\mathrm{y}$ & 1.456 & 0.73 & 743.71 & 9.91 & $\mathrm{n}$ & $22.950 \pm 0.044$ & $-0.23 \pm 0.11$ \\
\hline 23 & 55354.921213 & $\mathrm{y}$ & 1.539 & 0.86 & 743.74 & 10.15 & $\mathrm{n}$ & $22.984 \pm 0.058$ & $-0.15 \pm 0.09$ \\
\hline 24 & 55354.931026 & $\mathrm{y}$ & 1.640 & 1.18 & 743.79 & 9.98 & $\mathrm{n}$ & $22.890 \pm 0.034$ & $-0.16 \pm 0.09$ \\
\hline
\end{tabular}

Notes. ${ }^{(a)}$ Was the Image Slicer (IS) used? (Yes/No). ${ }^{(b)}$ Was the observation obtained during transit? (Yes/No).

\section{References}

Albrecht, S., Winn, J. N., Johnson, J. A., et al. 2011, ApJ, 738, 50 Alonso, R., Auvergne, M., Baglin, A., et al. 2008, A\&A, 482, L21 Ammler-von Eiff, M., Santos, N. C., Sousa, S. G., et al. 2009, A\&A, 507, 523

Assef, R. J., Gaudi, B. S., \& Stanek, K. Z. 2009, ApJ, 701, 1616

Beck, C. A. R., \& Rezaei, R. 2011, A\&A, 531, A173

Bouchy, F., Queloz, D., Deleuil, M., et al. 2008, A\&A, 482, L25

Charbonneau, D., Brown, T. M., Noyes, R. W., \& Gilliland, R. L. 2002, ApJ, 568,377

Claret, A. 2004, A\&A, 428, 1001

Czesla, S., Huber, K. F., Wolter, U., Schröter, S., \& Schmitt, J. H. M. M. 2009, A\&A, 505, 1277

Dekker, H., D’Odorico, S., Kaufer, A., Delabre, B., \& Kotzlowski, H. 2000, in SPIE Conf. Ser. 4008, ed. M. Iye, \& A. F. Moorwood, 534

Dreizler, S., Reiners, A., Homeier, D., \& Noll, M. 2009, A\&A, 499, 615

ESO Quality Control Group 2005, UVES - general pipeline information, http://www .eso.org/observing/dfo/quality/UVES/pipeline/

Fabrycky, D. C., \& Winn, J. N. 2009, ApJ, 696, 1230

Figueira, P., Pepe, F., Lovis, C., \& Mayor, M. 2010, A\&A, 515, A106

Gillon, M., Lanotte, A. A., Barman, T., et al. 2010, A\&A, 511, A3

Giménez, A. 2006, ApJ, 650, 408

Gray, R. O., \& Corbally, C. J. 1994, AJ, 107, 742

Hirano, T., Suto, Y., Taruya, A., et al. 2010, ApJ, 709, 458

Huber, K. F., Czesla, S., Wolter, U., \& Schmitt, J. H. M. M. 2009, A\&A, 508, 901

Huber, K. F., Czesla, S., Wolter, U., \& Schmitt, J. H. M. M. 2010, A\&A, 514, A39

Jones, E., Oliphant, T., Peterson, P., et al. 2001, SciPy: Open source scientific tools for Python, http: //www. scipy.org

Kass, R. E., \& Raftery, A. E. 1995, J. Am. Stat. Assoc., 90, 773

Kaufer, A., Dumas, C., \& Monaco, L. 2011, UV-Visual Echelle Spectrograph User manual, VLT-MAN-ESO-13200-1825, Issue 88.2

Knutson, H. A., Howard, A. W., \& Isaacson, H. 2010, ApJ, 720, 1569

Kurucz, R. L. 1993, SYNTHE spectrum synthesis programs and line data (Cambridge, MA: Smithsonian Astrophysical Observatory)
Lanza, A. F., Pagano, I., Leto, G., et al. 2009, A\&A, 493, 193

McLaughlin, D. B. 1924, ApJ, 60, 22

Newton, M. A., \& Raftery, A. E. 1994, J. R. Statist. Soc. B, 56, 3

Ohta, Y., Taruya, A., \& Suto, Y. 2005, ApJ, 622, 1118

Pál, A. 2008, MNRAS, 390, 281

Patil, A., Huard, D., \& Fonnesbeck, C. J. 2010, J. Stat. Soft., 35, 1

Pettit, E. 1946, PASP, 58, 310

Piskunov, N. E., \& Valenti, J. A. 2002, A\&A, 385, 1095

Piskunov, N. E., Kupka, F., Ryabchikova, T. A., Weiss, W. W., \& Jeffery, C. S. 1995, A\&AS, 112, 525

Radick, R. R., Lockwood, G. W., Skiff, B. A., \& Baliunas, S. L. 1998, ApJS, 118, 239

Rawlings, J. O., Pantula, S. G., \& Dickey, D. A. 1998, Applied regression analysis, 2nd edn., Springer texts in statistics (New York, NY: Springer)

Redfield, S., Endl, M., Cochran, W. D., \& Koesterke, L. 2008, ApJ, 673, L87

Reichart, D., Nysewander, M., Moran, J., et al. 2005, Nuovo Cimento C Geophysics Space Physics C, 28, 767

Reiners, A. 2009, A\&A, 498, 853

Rossiter, R. A. 1924, ApJ, 60, 15

Rothman, L. S., Gordon, I. E., Barbe, A., et al. 2009, J. Quant. Spec. Radiat. Transf., 110, 533

Schlawin, E., Agol, E., Walkowicz, L. M., Covey, K., \& Lloyd, J. P. 2010, ApJ, 722, L75

Schlesinger, F. 1910, Publications of the Allegheny Observatory of the University of Pittsburgh, 1, 123

Schröter, S., Czesla, S., Wolter, U., et al. 2011, A\&A, 532, A3

Seager, S., \& Sasselov, D. D. 2000, ApJ, 537, 916

Snellen, I. A. G. 2004, MNRAS, 353, L1

Snellen, I. A. G., de Mooij, E. J. W., \& Burrows, A. 2010, A\&A, 513, A76

Triaud, A. H. M. J., Queloz, D., Bouchy, F., et al. 2009, A\&A, 506, 377

Vidal-Madjar, A., Lecavelier des Etangs, A., Désert, J., et al. 2003, Nature, 422, 143

Wolter, U., \& Schmitt, J. H. M. M. 2005, A\&A, 435, L21

Zucker, S. 2003, MNRAS, 342, 1291 